\title{
Use of ozone in water, agriculture and zootechnics: relationships between dysbiosis and mental disorders
}

\author{
Giuseppe Vitali, ${ }^{1}$ Luigi Valdenassi ${ }^{1,2}$ \\ ${ }^{1}$ Italian Society for Oxygen-Ozone Therapy (SIOOT), Gorle (BG); ${ }^{2}$ University of Pavia, Pavia, Italy
}

\begin{abstract}
Ozone $\left(\mathrm{O}_{3}\right)$ is a bluish-coloured gas with a characteristic odour that forms in the layers of the atmosphere, near electric shocks, sparks or lightning; the extremely high voltages reached during thunderstorms produce ozone from oxygen. The particular fresh, clean odour, the smell of spring rain is the result of the ozone reproduced by nature. Ozone comes from the Greek word ozein, which means to sense the odour of. Ozone is an essential gas for life on Earth, allowing the absorption of ultraviolet light emanating from the Sun; in fact, the ozone layer in the stratosphere protects against the harmful action of UV-B ultraviolet rays. The gas, not being stable over the long term, is therefore not produced in cylinders; it can currently be prepared through special, certified and authorised devices, which use small electric discharges to convert the oxygen into ozone. It is a molecule formed by three oxygen atoms $\left(\mathrm{O}_{3}\right)$, with a negative electric charge. It has a short half-life, and will therefore decay after a certain time back to its original form: oxygen. Essentially ozone is nothing but oxygen $\left(\mathrm{O}_{2}\right)$ with an extra oxygen atom, which has a high electrical charge. Ozone works according to the principle of oxidation. The oxidation mechanism follows two paths: i) Direct: contact of the molecule with the contaminant; ii) Indirect: the ozone decomposes into hydroxyl radicals, more powerful but short-lived. Both reactions occur simultaneously.
\end{abstract}

Correspondence: Giuseppe Vitali, Italian Society for Oxygen-Ozone Therapy (SIOOT), via Don Luigi Sturzo 2, 24020 Gorle (BG), Italy. E-mail: info@ossigenoozono.it

Key words: Ozone; Dysbiosis; Mental disorders.

Contributions: the authors contributed equally.

Conflict of interest: the authors declare no potential conflict of interest.

Funding: none.

Received for publication: 1 March 2019.

Accepted for publication: 21 March 2019.

CCopyright G. Vitali and L. Valdenassi, 2019

Licensee PAGEPress, Italy

Ozone Therapy 2019; 4:8182

doi:10.4081/ozone.2019.8182

This article is distributed under the terms of the Creative Commons Attribution Noncommercial License (by-nc 4.0) which permits any noncommercial use, distribution, and reproduction in any medium, provided the original author(s) and source are credited.
When the static charged ozone molecule $\left(\mathrm{O}_{3}\right)$ comes into contact with something capable of oxidising, the ozone molecule's charge flows directly over it. This happens because ozone is very unstable and tends to change back into its original form $\left(\mathrm{O}_{2}\right)$. Ozone can oxidise with all kinds of materials, but also with odours and microorganisms such as bacteria, viruses and fungi. The supplemental oxygen atom is released from the ozone molecule and binds to the other material. In the end, only the pure and stable oxygen molecule remains. Ozone is one of the strongest oxidation techniques available for oxidising solutes. The supplemental/added oxygen atom will bind (= oxidation) in a second to each component that comes into contact with ozone. It is used for a wide range of purification processes. It can be employed for disinfection in municipal wastewater and in drinking water treatment plants. However, ozone is increasingly used in the industrial sector. In the food industry, for example, it is used for disinfection, and in the textile and paper industry it is used to oxidise wastewater. The main benefit of ozone is its clean nature, because it only oxidises the materials, barely forming any by-products. Since ozone has a strong characteristic distinctive odour, even very low concentrations can be quickly perceived. This generally makes it safe to work with. Since Chlorine is still the best-known oxidising and disinfectant agent, ozone is often compared with chlorine. Unlike chlorine, antibiotics or various chlorine derivatives that have no effect, ozone acts on viruses and spores. In its sterilising action, ozone directly attacks bacteria by inducing a catalytic oxidation process on the mass of bacterial proteins, unlike chlorine which acts only through specific enzymatic poisoning of vital centres, a process which requires a longer time interval and sensitive quantity for its diffusion inside the cytoplasm. Regarding the virucidal action, it is interesting to keep in mind that with a residual ozone rate of $0.6 \mathrm{ppm}$ (parts per million) and with a contact time of 2 minutes, the percentage of inactivation for bacteria and viruses present in the disinfection liquid is total. Ozone's oxidising power is 120 times greater than that of chlorine.

\section{Introduction}

It can be deduced that for $1 \mathrm{~g}$ of ozone, obtaining a similar bactericidal action with chlorine would take $120 \mathrm{~g}$, which would make the water difficult to use.

It is worth remembering that chlorine in significant quantities is carcinogenic and should not be used in high concentrations for a long time.

Directive 98/83/EC of 03/11/1998 no longer indicates chlorine as a disinfectant agent, as it is unable to meet the chemical-physical and bacteriological values in the standard.

Ozone is the only method able to comply with the requirements of Directive 98/83/EC (Table 1). 
To date, based on current legislation on the matter, the water from aqueducts still has chlorine added, the limits of which are highlighted in the table above.

From this it is important to note ozone's sterilisation abilities against viruses and bacteria that may be present in the water we normally take in for food purposes.

An important characteristic of ozone is that in the course of its action it does not give rise to negative additional or substitute products, but develops beneficial oxygen. Unlike chlorine, ozone does not produce appreciable residues at the end of its sterilisation action.

Ozone, even if dosed in excess, after about 5 minutes of contact is completely transformed into oxygen, with a significant enrichment of oxygen in the water. In fact the water normally has 20/25 parts of oxygen, while with this treatment $80 / 120$ parts of oxygen are obtained. For purifying water and air, it is necessary to produce ozone on site. Due to its short half-life, the ozone decays soon after being produced. The half-life of ozone in water is about 30 minutes, which means that every half hour its concentration is reduced by half compared to the initial concentration. For example when you have $8 \mathrm{~g} / 1$, the concentration is reduced every 30 minutes as follows: $8 ; 4$; $2 ; 1$; etc. In practice the half-life is shorter because it can be influenced by many factors. These factors are temperature, $\mathrm{pH}$, concentration and type of solutes. Since ozone reacts with all types of components, its concentration will be reduced rapidly (Table 2).

Thanks to its oxidising power, ozone is used to: i) disinfect; ii) sanitise; iii) deodorise; iv) fulfil various uses in the healing field.

In destroying algae and bacteria, inactivating viruses and oxidising many organic and inorganic contaminants present both in air and in water, it can therefore be used in various sectors and environments: i) hospitals; ii) rest homes; iii) schools; iv) kitchens (canteens, restaurants, hotels); v) sports centres; vi) industry; vii) Food laboratories; viii) Butchers; ix) Fishmongers.

The industrial uses of ozone include: i) disinfecting surfaces intended for contact with food; ii) disinfecting meat from mould and yeast spores; iii) disinfecting fish from mould spores and bacteria; iv) disinfecting fruit and vegetables from mould and yeast spores; v) oxidising chemical water pollutants (iron, arsenic, hydrogen sulphide, nitrites and organic complexes); vi) aiding the flocculation of sludge active in water purification; vii) cleaning and bleaching fabrics; viii) disinfecting public areas; ix) disinfecting and deodorising in craft activities; $\mathrm{x}$ ) disinfecting and deodorising all hotel and restaurant environments, rest homes, schools, cinemas, etc; xi) disinfecting water in aqueducts; xii) disinfecting water in swimming pools; xiii) disinfecting water destined for bottling.

The application of ozone in treating drinking water. The typical scheme of a purification plant for traditional surface water is as follows: i) pre-oxidation with chlorine; ii) flocculation with chemical agents; iii) sedimentation; iv) filtration; v) accumulation; vi) final disinfection again with chlorine.

Potential problems: i) insufficient removal of organic compounds; ii) formation of trihalomethanes with the oxidation of organic material; iii) presence of taste and odour; iv) no removal of algal toxins; v) inadequate disinfection: Protozoa such as Cryptosporidium, Giardia Lamblia (cyst-resistant forms). The water does not comply with legislative limits.

The scheme of a purification plant for surface water with ozone is composed as follows: i) pre-oxidation with ozone; ii) flocculation with chemicals; iii) sedimentation; iv) intermediate oxidation with ozone; v) sand filtration; vi) post-oxidation with ozone; vii) granular activated carbon (GAC) or sand filtration; viii) accumulation; ix) final disinfection with $\mathrm{UV}$; $\mathrm{x}$ ) disinfection of network coverage.

The use of ozone leads to:

i) Improved coagulation, ozone produces the polarisation of the molecules increasing aggregation capacity, improves sedimentation, reduces the presence of organic compounds, leads to savings in the use of chemicals and in management.

ii) Oxidation of iron and manganese: ozone transforms soluble salts into insoluble oxides or hydroxides, the hydroxides and metals are easily removed through sedimentation, organic compounds bound to iron and manganese are not oxidised with air or oxygen, chlorine does not oxidise manganese.

iii) Taste and odour control: the presence of flavours and odours in water is often due to the chlorination of natural organic compounds such as phenols, hydrogen sulphides, algae, etc. Chlorine + phenols $=$ flavour + toxic compounds, with the oxidation of organic compounds with ozone: no taste and odour, no toxic compound.

iv) Oxidation precursory to the formation of trihalomethanes, ozone destroys the organic compounds precursory to the formation of trihalomethanes (Resorcinol, Aniline, Phenols and Polyphenols, Benzoic Acid, Salicylic Aldehyde), ozone destroys the precursors.

v) Algae and By-products, algae can be treated in two steps, in pre-oxidation before flotation or filtration by direct action, or in intermediate ozonation, ozone reduces toxins, taste and odours produced by algae.

vi) Elimination of Nitrites in groundwater, ozone transforms $\mathrm{NO} 2$ (Nitrites) into NO3 (Nitrates) in pre- or inter-ozonation.

vii) Oxidation of sulphides, in subsoil waters, often responsible for bad odours, smell of rotten eggs.

In combination with sand or GAC filtration, ozone has the ability to procure organic material reduction, colour removal, pesticide reduction and disinfection, and significantly reduce many other micropollutants. These are substances present in low concentrations that are difficult or impossible to remove with conventional treat-

Table 1. Characteristics of ozone.

\begin{tabular}{lcc}
\hline Action & Chlorine & Orone \\
Odour & $\begin{array}{c}\text { Unpleasant } \\
\text { Colour }\end{array}$ & None \\
\hline Antiviral activity & Nending towards yellow & None \\
Antibacterial activity & Variable & High \\
\hline Destructive activity on algae and protozoa & Slight & High \\
Destructive activity on fungi & Slight & High \\
\hline Destructive activity on spores and cysts & Slight & High \\
Activity on organic molecules & None & High \\
\hline
\end{tabular}

Table 2. Resistance of ozone in water based on temperature.

\begin{tabular}{lccc} 
Temperature & Days & Hours & Minutes \\
$40^{\circ} \mathrm{C}$ & 0 & 0 & 32 \\
$35^{\circ} \mathrm{C}$ & 0 & 1 & 5 \\
\hline $30^{\circ} \mathrm{C}$ & 0 & 2 & 12 \\
$25^{\circ} \mathrm{C}$ & 0 & 4 & 32 \\
\hline $20^{\circ} \mathrm{C}$ & 0 & 10 & 0 \\
$15^{\circ} \mathrm{C}$ & 0 & 22 & 9 \\
\hline $10^{\circ} \mathrm{C}$ & 2 & 2 & 0 \\
$5^{\circ} \mathrm{C}$ & 4 & 22 & 0 \\
\hline $0^{\circ} \mathrm{C}$ & 11 & 22 & 0
\end{tabular}


ment technologies. Substances that relate to hormonal functions, and which can be for example fragrances, sun protection creams, beauty or cleaning products, pharmaceuticals and personal care products. The presence of these micropollutants has a negative impact on our ecosystem, on fish, with evident phenomena of effects on male sexuality and therefore a decrease in male fertility, an impact on humans too, with a reduction in fertility, and an increase in tumour incidence. The use of ozone in drinking water treatment plants to obtain a safe result is very brief: depending on the situation, the contact time can range from 2 minutes to a maximum of 10 minutes, with dosages ranging from 0.5 to $5 \mathrm{gO} 3 / \mathrm{m} 3$. Among ozone's many merits is also that of not being dangerous to humans and pets if exposed for short periods, as within minutes of being produced it decomposes completely, turning back into oxygen.

Ozone is produced by means of Multiossigen generators, suitable electronic equipment that allows the creation of gas through controlled electrical discharges.

The Multiossigen generator takes oxygen, composed of two $\mathrm{O}_{2}$ atoms, and joins it with a third oxygen atom, forming the ozone molecule $\left(\mathrm{O}_{3}\right)$.

There are no residues to dispose of and the treatment can be properly controlled whenever wanted.

Ozone is a gas that penetrates everywhere, which is why, if produced in adequate quantities, it can be defined as having global coverage.

The reliable advantages of using ozone can be summarised in the following points: i) oxidising power; ii) it does not cause secondary pollution, (after the reaction, the ozone degrades to molecular oxygen and does not leave harmful residues); iii) it degrades pollutants, without transferring the pollution to other phases; iv) the strong disinfection and oxygenation avoid corrosive and fermentative phenomena with consequent odour emissions, even in the case of prolonged stops; v) great dosing flexibility and simple installation (which minimise costs for management and operative control); vi) it sanitises and deodorises environments without using chemicals and without leaving any residue; vii) it does not produce sludge or concentrates; viii) it improves the general characteristics of water and increases the biodegradability of wastewater; ix) after ozone treatment the wastewater is already disinfected, thus avoiding the use of chlorine compounds and therefore the formation of toxic organic by-products. ${ }^{1-20}$

\section{Microbial properties of ozone}

While in developing countries Typhoidal Salmonella and Cholera Vibrios are typical bacteria transmitted by water, in developed countries this can represent a vehicle for Salmonella Typhimurium, for some types of vibrios such as Vibrio Vulnificus, e.g. Aeromonas.

Hydrophilus, also present in alpine waters, and Legionella Pneumophila, also present in volcanic waters $\left(45^{\circ}-50^{\circ}\right)$ and in air conditioning systems.

In surface waters cyanobacteria, producers of entero- and neurotoxin, are relatively frequent, not to mention dozens of other bacteria, viruses and protozoa that often combine with each other in biofilms, and which in turn protect the microorganisms that compose them.

Disinfection means purification of the medium until it becomes incapable of causing infectious diseases in humans, animals and plants that come into contact with the medium (especially water).

On various germs, ozone is one of the most effective disinfectants, acting in concentrations equal to fractions of ppm and in a short time.

Because of its high oxidising potential, ozone oxidises the cellular components of the bacterial cell wall by penetrating into the cell. Once inside, it oxidises all the essential components (enzymes, proteins, DNA, RNA).

During this process the membrane is damaged and the cell dies.

\section{Sporicidal, fungicidal and protozoicidal properties}

As well as its bactericidal and virucidal activity, ozone has sporicidal, fungicidal and protozoicidal properties.

As a sporicide, ozone does not only have scientific interest; since the early 1980s ozone, recognised as a safe substance to use, has been approved as a disinfectant to be added to bottled water, then to water used for freezing poultry, storing eggs, etc. all processes for which spore inactivation is essential.

In-depth research has demonstrated the gas' effectiveness on spore-forming bacilli such as:

B. Cereus, B. Megaterium, C. Clostridi, etc.

Finally, as regards ozone's protozoicidal effect, we recall that in Europe the most well-known diseases due to these parasites are: Amebiasis, Toxoplasmosis and Malaria.

Entamoeba Histolytica is not the most sensitive protozoan to ozone, although the active percentages of the gas remain rather low; on the other hand, this protozoan is also relatively resistant to chlorine as shown by some epidemics, such as the one that occurred many years ago in Chicago, where water (badly) treated with chlorine was used.

The specific activity of ozone on amoebas is nowadays of great relevance in preventing nosocomial pneumonia from Legionella Pneumophila, a germ that develops most commonly in hot water. While intestinal infections due to water contaminated with Giardia are well known, those caused by Cryptosporidium are less known, although in recent times, in addition to single cases of gastrointestinal infections, it has caused dozens of epidemics.

Both Giardia and Cryptosporidium are present in the waters of all the world's rivers. Since common filtrations cannot guarantee the elimination of protozoa, we recall that ozone is active against Cryptosporidium at very low concentrations and in a short time, while Giardia is sensitive to even lower ozone concentrations.

In water, therefore, there are many pathogenic microorganisms, such as bacteria, viruses and protozoa. Bacteria are singlecelled organisms of sizes ranging from 0.1 to 10 microns. The most commonly known species are E. Coli and Salmonella.

Viruses are a very small group that can cause infections. They are found in animals, plants and humans at the intracellular level. Viruses differ from bacteria in that they have no cytoplasm or cellular organelles. A virus or virion particle is just a small lump of genetic material (DNA or RNA) surrounded by a blanket of proteins. There are only a few types that contain a cell membrane. The size of a virus can be between 0.01 and 0.1 microns. Examples of commonly known viruses are Rotavirus and the pneumonia virus. Protozoa are single-celled organisms that contain a nucleus. They are very mobile and feed on solid nutrients. They can be found in water, where they are a source of food for fish and other aquatic animals. Protozoa vary in size from 1 to 20 microns. Some examples of protozoa are Cryptosporidium and Giardia Lambia.

For most disinfectants, protozoa are the most resistant, followed by viruses; bacteria rarely resist disinfection.

Protozoa have caused many epidemics in the United States and Great Britain through drinking water. In many cases, the drinking water met bacteriological quality standards. As a result of the resistance of Cryptosporidium and Giardia in the environment and their resistance to disinfectants, the direct purification of surface water was in accordance with very strict quality requirements.

Protozoa spread quickly through the environment as oocysts (spores), which live longer and are more resistant to disinfectants than bacteria or viruses. In general, Cryptosporidium causes more 
problems for the treatment of drinking water than Giardia, as the former has a size of 4-5 microns which makes it difficult to remove by conventional filtration, while the latter has a size of 8-14 microns, which makes it easier to remove by conventional filtration.

Furthermore, when pool water or drinking water is treated with conventional chlorination, Cryptosporidium is insufficiently deactivated.

Alternative treatment methods, such as ozone and UV disinfection, seem to have the ability to sufficiently inactivate the oocysts.

As mentioned previously, the oxidising power of ozone is 120 times that of chlorine, and is used to neutralise exhaust gases containing sulphate; or in sports environments to sanitise spaces (gyms, locker rooms, bathrooms, communal rooms), or even in sports cosmetics (massage products and creams); or in spa treatments (such as balneotherapy, which treats venous and lymphatic insufficiency).

In our tap water, defined as potable, and which is certainly frequently checked, concentrations of arsenic, ammonia, chromium, pesticides, chemical fertilisers and many other not particularly healthy substances are allowed by law, in non-excessive doses.

This water is also chlorinated to eliminate bacteria, but has no effect on viruses and other pollutants.

Chlorinated water destroys vitamin E, which is very important as an antioxidant and specifically for the health and well-being of the skin.

Chlorine is derived from sodium hypochlorite, the same one used to make bleach. The quantity that goes in is different, but nevertheless highly carcinogenic over time. These toxins also accumulate within our cells day after day.

Bottled mineral water is extracted from aquifers, and if these are polluted, then to a different extent so is the mineral water.

The springs it is taken from receive water from the rain: it does not have a preferential route, avoiding pollution of rivers and land, and considering the large quantity of chemical waste produced by our consumer society, most of which inevitably ends up in the water, it is very likely that many elements can be found in the bottles which are certainly not healthy.

The bottles, when filled, should not be exposed to the sun, because with the heat the bacterial quantities that may already be present increase in number.

Our body is mostly composed of water. All our organs depend on water and contain it in large quantities. We can really say that without water there is no life, but at the same time that the quality of life depends on the quality of the ingested water.

Consider that the amount of water present in our body ranges from an average of $75 \%$ in a young child to around $50 \%$ in an elderly person.

After all, it is clear: in adults and the elderly, the skin is drier and less elastic, the tissues are less smooth and tense, the sign of pressure exerted on the skin by the thumb remains visible for longer. The biological age of the skin of the whole organism is easily determined by its state of hydration. Even the bones contain water, although to a much lesser extent than the soft tissues.

$40 \%$ to $50 \%$ of our body water is contained within the cells, and constitutes the so-called intracellular fluid. The remaining $20 \%$ is found in the interstices between the cells: this is the extracellular fluid.

There is an abundance of potassium in the intracellular environment and sodium in the extracellular environment. The correct balance of this natural stock is indispensable for life and for the correct performance of metabolic functions.

Water is by nature a solvent. It has the function of dissolving and transporting the main nutrients in all the cells, promoting digestion, ensuring thermoregulation (including through impercep- tible sweating), and transporting waste out of our organism through the excretory organs.

Water also acts as a shock absorber for the most delicate organs such as the eye, the inner ear and the brain.

The vital functions we have listed here probably do not even represent the total number, due to the fact that some of the very important activities carried out by water are still only half-known: that of acting as an antenna or information transmitter, or that of being a kind of reservoir of memory or battery capable of recharging our vital energies. In short, the water we drink does not only quench our thirst or refresh us, but rather carries out a large number of irreplaceable functions, which in some ways are still unknown.

Let us reflect only on this: the human body is immersed in water. The body of an adult, in which the presence of water is around $65 \%$, will contain $40-50$ litres.

Due to reduced ability to sort sensory impulses, in old age the feeling of thirst also decreases.

If the water content in the cell falls below $50 \%$, vital processes are paralysed, often irreversibly.

During his lifetime, a man drinks about 25,000 litres of water.

It is easy, then, to understand the importance of water, both from the quantitative and qualitative point of view, for the perfect functioning of the human organism.

We can therefore reiterate that water is the main component of the human body and that not all water is the same.

Here then are the fundamental reasons for drinking a lot of water that is truly pure and rich in oxygen: dehydration, even of 1$2 \%$ of body weight, decreases our strength and makes us feel tired.

It is essential for the proper functioning of our digestive system, the cardio-circulatory system and all the other organs and systems. In 2002, a study stated the thesis that a good water supply decreases the risk of a cardiac event by about $41 \%$.

Reduced intake can be a cause of headaches.

The amount of water it is necessary to drink in normal conditions can be calculated using the following formula: weight in $\mathrm{kg}$ $\times 3: 100$. Example: $70 \times 3=210: 100=2.1$ litres of water needed.

Drinking impure and inadequately oxygenated water can cause toxic substances to build up inside our cells, which can cause a lot of damage: i) liver deposits and poisoning; ii) renal and biliary calculi; iii) vascular problems; iv) cellulite; v) headaches; vi) weight increase; vii) intestinal dysmetabolism (dysbiosis).

The FDA (the U.S. Ministry of Health) currently certifies that ozone is the best agent capable of destroying all pathogenic germs in water purification, and simultaneously also destroys all pollutants. Free of toxic elements, of bacterial and viral charges, it has an appropriate magnetic polarisation, which means re-establishing correct cell polarisation with the restoration of intra- and extracellular exchanges.

Daily use of hyperozonated water is widely employed in treating numerous gastric diseases such as helicobacter pylori gastritis, eliminating the bacterium after about fifteen days of taking hyperozonated water, thus avoiding the use of antibiotics.

Oral intake of two to four glasses a day is a very effective treatment for: i) colitis and diseases of the large intestine; ii) chronic cystitis; iii) intestinal dysbiosis; iv) irritable colon, colon cleansing (rectal irrigation); v) helicobacter; vi) gastric reflux; vii) acidosis; viii) rhinitis (endonasal washes); ix) vaginitis (vaginal lavage); $x$ ) halitosis; xi) gingivitis, mouth ulcers; xii) inflammation of the eye (periocular washes); xiii) cellulite and overweightness; xiv) conditions of oxygen deficiency; xv) increases immunity levels and defences against most diseases.

A healthy organism has a normal Microflora, which not only constitutes a barrier to infections, but also has other useful and necessary functions for maintaining good health. 
It is important for the antagonistic defence function against pathogenic germs and harmful bacteria, thanks to the production of natural antibiotics and bacteriocins.

We define Eubiosis as the situation in which there is a balance between the intestinal flora and the organism; in this case the symbiosis becomes advantageous to both.

Human health is regulated by the superorganism represented by the intestinal microbiota, which is the entire bacterial population that occupies the central part of the human digestive system, consisting of about 3 million genes which have existed for 5 billion years.

The microbiota is in fact composed of a number of bacteria 10 times the total number of cells that make up the entire human body, and of more or less 4 million different types of bacteria.

The intestinal microbiota, ultimately, represents a world within us whose main task is to perform various functions, ranging from metabolising certain nutrients to defending the body, from synthesising vitamins to regulating the immune system's activity.

Dysbiosis, on the other hand, is defined as when there is an alteration of this microbial ecosystem, with disturbance and gradual alteration of the normal bacterial flora.

We talk about intestinal dysbiosis to indicate a set of symptoms and functional gastrointestinal disorders that can evolve, involving organs and systems far from the colon, through microbial lymphogenic seeding with blocking of mucosa-associated lymphoid tissue (MALT) - a widespread lymphoid tissue at the level of the mucous membranes such as the gastro-intestinal tract, the urogenital tract, the thyroid, the lungs, the eyes and the skin; these formations are not organised to form organs of the lymphatic system but rather as lymphatic nodules or isolated cells - and gut-associated lymphoid tissue (GALT) - the part of the immune system present in the digestive tract. GALT is an example of lymphoid tissue associated with the mucosa, which is responsible for protecting the mucous membranes from attacks by pathogens, both in the primary and secondary response.

The gastrointestinal system represents a way of communication with the external environment and is largely populated by potentially pathogenic microorganisms (especially the intestine), and therefore a strong presence of the immune system at the level of the mucous membranes is necessary to ensure control of these populations.

In the intestine there are between 100 and 200 lymphatic stations (Peyer's patches) equal to $50 / 80 \%$ of our immune system, so the failure of this defensive system has very serious consequences. We also recall that there is a strong static increase in serious colon diseases (diverticula, polyps, ulcerative colitis, tumours, etc.).

Therefore, for a whole series of reasons, this good intestinal flora, formed by billions of bacteria that live in symbiosis with our organism, carrying out tasks fundamental to our health (e.g. producing B vitamins), is gradually altered and destroyed, and replaced by other bad bacteria, which better adapt to an unbalanced gut condition.

These bacteria, mostly anaerobes (fungi, moulds, candida, etc.), survive and duplicate in the absence of oxygen, produce air formation, fermentation, putrefaction and toxic substances (ammonia, phenol, cadaverine, putrescine, etc.) that are reabsorbed by the capillaries, causing poisoning of the whole organism.

Dysbiosis currently represents a social problem affecting between $30 \%$ and $60 \%$ of patients who undergo gastroenterological examinations, very frequently and significantly caused by functional alteration of the colon's mucous membrane and changes to the intestinal bacterial flora, with consequent alteration of the latter's composition.

The key role of intestinal microflora in the gastrointestinal ecosystem is primarily in anti-infective immune modulation, secondly in inflammatory modulation (cytokines), and lastly in autoimmune responses and allergies.

The intestinal epithelium is the most extensive and most critical interface between the organism and its environment; it has an extension with microvilli of 2000 square metres and has the function of a first barrier.

If we consider the vast surface of the gastrointestinal mucosa and its great absorption capacity, it will be understood that an alteration of the intestinal conditions generates many toxic substances (toxins) which, after being released into the circulation through the blood and lymphatic pathways, are distributed in various parts of the body, creating multiple dysfunctions and pathologies.

Many people suffer from it, displaying many of these symptoms: i) excess gas and flatulence; ii) indigestion; iii) halitosis; iv) delay of faecal transit with abdominal spasms; v) decreased absorption of water and salt with diarrhoea; vi) excessive absorption of water and consequent constipation; vii) conditions involving alteration of the dermis (acne, cellulite, herpes, etc.); viii) metabolic alterations; ix) mood changes; $x$ ) sleep disorders; xi) asthenia; xii) headaches; xiii) allergies; xiv) vaginal candidiasis; xv) dysmenorrhea.

There are many and varied reasons why this delicate balance is altered: i) food-related: intolerances, diets lacking in fibre or vegetables, excess sugar, alcohol; ii) iatrogenic: drugs (cortisone, painkillers, anti-inflammatories, antibiotics, laxatives, immunosuppressants, radiotherapy, oral contraceptives, psychotropic drugs such as antidepressants and anxiolytics); iii) smoking; iv) work environment; v) irregular lifestyles; vi) pollutants: dyes, preservatives, pesticides, heavy metals such as aluminium, mercury, lead, cadmium; vii) pathological: hepatopathies, chronic pancreatitis, intestinal infections, musculoskeletal pains; viii) neurogenic: prolonged stress, psychic traumas that act through immunological and endocrine mechanisms; ix) paediatric: immunity of gastrointestinal mechanisms.

We can have different types of dysbiosis:

i) Fermentation dysbiosis: mainly affects the stomach and small intestine, and is caused by a diet that is too rich in carbohydrates. The main symptoms are poor digestion, abdominal bloating, meteorism and flatulence.

ii) Putrefactive disease: typical of western populations, a diet that is too rich in protein (diet rich in fat and meat and low in fibre). It mainly affects the colon. The main symptom is constipation.

iii) Fungal disease (candida and excess yeast): a variant of fermentation dysbiosis, is due to the overgrowth of saccharomycetes or candida; promoted by a diet rich in sugars and low in fibre. The main symptoms are diarrhoea, bloating, anal itching, asthenia, allergy to antibiotics or other substances, hives, abdominal cystitis, mycosis in other areas. Food intolerances: yeast, bread, milk and derivatives, beer, and in part cereals. Diagnosis: evidence in faeces or other places, agglutination test, IgE anti-candida, IgE anti-yeast. Antibiotics make the situation worse.

iv) Sensitisation dysbiosis: an abnormal immune response to components of physiological microflora can contribute to the pathogenesis of: IBD (inflammatory bowel disease, chronic intestinal inflammatory diseases, are a group of nosological entities characterised by the presence of chronic inflammation in the absence of infectious aetiology. The two most important ones in this group are Crohn's disease and ulcerative rectocolitis; spondyloarthropathy and other connective diseases or dermatological diseases such as Psoriasis or Acne.

Laboratory data: alterations of transaminases and alpha-amylases, stool test, faecal $\mathrm{pH}$ (in the case of normal intestinal flora, the $\mathrm{pH}$ of the large intestine corresponds to a value between 5.5 
and 6.0 from infancy to adulthood. Values higher than $\mathrm{pH} 6.0$ are already suspect); the indole test on urine (urine normally contains small amounts of indole equal to $4-20 \mathrm{mg}$ in 24 hours). In the case of severe intestinal dysbiosis, skatole is also present in the urine.

As regards the therapeutic approach to the pathology, it must be remembered that the complexity of the pathogenetic and physiopathological phenomena that characterise dysbiosis must necessarily involve, from the therapeutic point of view, an integrated intervention that aims, on the one hand, to act on the host, and on the other hand, at homeostatic balance of the intestinal microclimate and restoring the balance between the different bacterial species.

Treatment for intestinal dysbiosis must focus on changing eating habits:

i) Eating fruit separately from meals;

ii) Eating meals at the same time;

iii) Drinking at least 1.5 litres of water per day (increasing the quantity in summer);

iv) Respecting the correct food associations (raw and cooked vegetables must be associated with cereals, or legumes, or protein concentrates such as seitan, tofu, soy, etc.)

v) Consuming only one type of food at a time (especially protein) in the same meal;

vi) Eating enough, neither too much nor too little (the stomach must be $2 / 3$ full. $1 / 3$ must be free from food for effective peristaltic activity. Gastric fullness is not synonymous with nutrition);

vii) Not eating food after 10:00 pm (the $\mathrm{pH}$ drops considerably during the evening and night)

All cheeses and derivatives are the most acidifying foods par excellence, followed by animal proteins and refined grains and derivatives; conversely, the most alkalising foods are fruit and all vegetables and greens.

Acidifying foods divided by group are shown in descending order:

A) Dairy products and derivatives: grana cheese, mozzarella, pecorino, emmental, camembert, eggs, soft fat cheese, whole yoghurt, pasteurised whole milk, ice cream.

B) Animal protein: canned beef, salami, boiled trout, beef steak, chicken, lean pork, cod fillet, sausages.

C) Refined flours and cereals.

D) Fruit, vegetables and greens in general are by far the most alkalising.

So taking into account their glycaemic index, during the day the choice of foods should follow the following indications: i) favour foods with a medium-high glycaemic index in the morning; ii) avoid foods with a high glycaemic index after 2:00 pm; iii) foods with a medium glycaemic index and low glycaemic load are allowed until 4:00 pm; iv) after 4:00 pm, only foods with a low glycaemic index, low glycaemic load and with low or medium acidifying power are allowed.

All this must be combined with a factor of fundamental importance: that of restoring the correct balance of bacterial flora through an appropriate combined treatment. The latter would have two functions, that of draining the gastrointestinal system and its functionally related organs (liver, pancreas, etc.), and preparing the colon for subsequent recolonising treatment with a mixture of oxygen-ozone and probiotics. In recent years, ozone therapy has proved to be a medical treatment with broad possibilities for application in many pathologies of the human body, and not only that, but also in many other fields. Its efficacy has been demonstrated for functional disorders of the colon and rehabilitation of intestinal peristaltic capacity (by rectal insufflation and above all hyperozonated water per os).
The therapy is well-tolerated by patients and does not present any side effects in the short or long term. Ozone therapy by rectal insufflation and hyperozonated water per os restores functionality to the intestine, with all the prerogatives for valid defence against bacteria, viruses and any other poisoning or dysfunction.

Ozonated water is widely used for various gastric pathologies such as Helicobacter Pylori gastritis, which become negative after about 30 days of therapy, thus avoiding the use of antibiotics, plus colitis, chronic diseases of the large intestine, and duodenitis.

Ozonated water is used to cleanse skin ulcers, in dentistry for pyorrhea and gum retractions and for buccal hygiene, stomatitis and paradontosis. In ophthalmology for treating conjunctivitis. In cosmetics to revitalise the skin.

Ozonated water has an antioxidant effect, thus succeeding in removing many invaders, including streptococci which cause pain. Ideal for bladder rinses in chronic cystitis and for vaginal washes in cases of infection. Ozonated water can be used to treat wounds and fungi.

It is worth highlighting the results obtained from work carried out from January 2012 to June 2013 by Dr. Fortunato Lo Prete and Dr. Francesco Vaiano.

The aim of the study, which lasted a total of 90 days, was to verify the effects induced by ozonated water and rectal insufflation on the overall state of well-being of 34 subjects suffering from intestinal dysbiosis.

The authors highlight how the $\mathrm{O}_{2}-\mathrm{O}_{3}$ mixture is a valid aid for restoring the physiological status of the altered mucous membranes, thus ensuring again the digestive and detoxifying functions of the gastrointestinal tract.

At the beginning, after 45 days and at the end of the study, at 90 days, some of the typical subjective symptoms of the treated pathology were assessed (chronic asthenia, meteorism, halitosis, alternate bowel constipation alternating with diarrhoea, poor digestion, gastralgia, irritable colon, aerophagia and troublesome belching).

The diagnosis was made by dosing two metabolites, indicating (dosed with the colorimetric method) and skatole (using the chromatographic method), in samples of biological fluids (urine).

The use of ozone in medicine is dictated by two general considerations, one based on the fact that ozone has direct and indirect effects on metabolism, and the other on the fact that it has biological effects.

\section{Effects of ozone on metabolisms}

i) Acceleration in the use of glucose by cells due to increased glycolysis, which increases the availability of ATP in the cells and therefore in the tissues, especially nervous tissue.

ii) Intervention on protein metabolism due to its affinity with sulphydryl groups, thus reacting with essential amino acids such as methionine and tryptophan or with cysteine which contains sulphur.

iii) Direct reaction with unsaturated fatty acids which are transformed into water-soluble compounds.

\section{Biological effects of ozone}

i) In the topical application there is disinfection and direct trophic action.

ii) For the formation of peroxides there is a systematic antibacterial and antiviral effect. The antiseptic mechanism is similar to that which the body usually uses in the formation, by leucocytes used for bacterial phagocytosis, of a molecule with antioxidant properties, similar to that of $\mathrm{O}_{3}$, i.e. $\mathrm{H}_{2} \mathrm{O}_{2}$. The germicidal effect of ozone depends above all on the presence of water and low temperatures. It also has a great ability to inactivate viruses (virusta- 
tic action), making them unable to adhere to cellular receptors on the target cell and then to replicate themselves.

While ozone has a virustatic action for viruses, for bacteria and in particular gram + it has a bactericidal and above all direct action.

iii) At the level of the red blood cells, reducing the global blood viscosity causes an increase in both their deformability and 2.3-diphosphoglycerate (responsible for the release of $\mathrm{O}_{2}$ by haemoglobin to the tissues). This has, as its ultimate goal, a clear improvement of $\mathrm{O}_{2}$ transport and therefore a rheological action.

\section{Effects of peroxides produced by ozone in phagocytosis}

During chronic infections the normal defensive processes are no longer able to destroy bacteria, due to insufficient formation of $\mathrm{H}_{2} \mathrm{O}_{2}$. It is at this level that I highlight the positive influence of peroxides formed during ozone treatment.

\section{Influence of ozone in the metabolism of erythrocytes}

Peroxide formation facilitates direct activation of erythrocyte metabolism. The first step of the reaction consists in the ozone's interaction with the double bonds of the unsaturated fatty acids of the phospholipid layer in the erythrocyte membrane. With the intervention of the glutathione system, an activation of glycolysis takes place, which leads to an increase of 2,3-diphosphoglycerate, with relative facilitation of the release of $\mathrm{O}_{2}$ in tissues by haemoglobin.

\section{Materials and Methods}

The study was conducted on adults: 34 subjects were considered, without age and sex limits, including 23 females (67.6\%) and 11 males (32.4\%), aged between 27 and 62 years old (average: 43.9).

The hyperozonated water, produced by means of the OM3 ozonator (from the Multiossigen company) was administered in the quantity of 3 glasses of $125 \mathrm{ml}$ each pro die.

Rectal insufflation was performed 3 times a week; the concentration of ozone (produced with the Multiossigen Medical 95 device) was $40 \mathrm{microg} / \mathrm{ml}$, while the total quantity of the $\mathrm{O}_{2}-\mathrm{O}_{3}$ mixture was $250 \mathrm{cc}$ per session; the total concentration of ozone administered was 10,000 micrograms.

All patients were adequately informed about the use of the treatment method for controlling some typical symptoms of intestinal dysbiosis, and gave their consent for this type of therapy.

For admission to treatment, adult patients were examined who met the following inclusion criteria: adults (age: 18-70), males and females with intestinal dysbiosis, not severe, confirmed with clinical and instrumental examination, competent to give consent to the study after receiving adequate information, able to guarantee sufficient adherence to prescription therapy and motivated to stay with this study until completion.

All patients underwent an initial descriptive pain assessment and a pain threshold measurement, to exclude subjects with evident hyperalgesia.

For the evaluation of oppressive abdominal pain and discomfort caused by other disorders typical of dysbiosis, an analogicalvisual scale was chosen, according to the Scott and Huskisson model, considered a subjective measure with a single dimension, since it evaluates only one component of the pain at a time.

The analogical-visual scale is the visual representation of the extent of pain that a patient thinks they feel. The extent can take the forms of both a pain scale and a pain relief scale.
The answers to the questionnaire seem potentially influenced by factors concerning the patient's psychophysical condition.

The optimal length to measure pain appears to be $10 \mathrm{~cm}$.

One end indicates the absence of pain, while the other represents the worst pain imaginable. The scale was compiled by the patients, who were asked to draw on the line a sign representing the level of pain experienced. The distance measured in numerical value, starting from the end indicating the absence of pain, represented the magnitude of the pain, represented the magnitude of the pain experienced, at that moment, by the patient.

The analogical-visual scale was given to patients undergoing treatment using a mixture of oxygen and nitrogen, at the beginning of the recruitment (before treatment), after 45 days and at the end of treatment after 90 days, asking them to mark, on the line between the two extremes, the magnitude of pain or symptoms taken into consideration, compared to the previous evaluation.

The fundamental criterion for evaluating the effectiveness of the treatment consisted in the percentage of patients who, at the end of the treatment, had a significant reduction in the values of the symptomatology taken into consideration, equal to at least $10 \%$ of the baseline value.

\section{Results}

The characteristics of the subjects treated are summarised in the tables, in time 0 , at 45 and at 90 days, at the end of the treatment.

The data, which was certainly interesting, was the clear and certainly significant improvement from the statistical point of view of constipation, meteorism, abdominal pain, gastralgia and asthenia, evaluated by means of the analogical-visual scale, according to the Scott and Huskinsson model, in subjects treated with $\mathrm{O}_{2}-\mathrm{O}_{3}$ mixture.

\section{Discussion and Conclusions}

All subjects displayed a significant improvement even at the first check, which was decidedly more significant, from a statistical point of view, at the end of the study.

The results of the study made it possible to verify, first of all, that intestinal dysbiosis is a condition with a greater incidence than what the clinic lets us establish on the basis of subjective and objective symptoms.

On the basis of the results obtained, in terms of improvement of the parameters considered and the correlated symptomatology, the association between rectal insufflation of the oxygen-ozone mixture with the oral intake of hyperozonated water recorded quite encouraging results, to the point where the authors could conclude that this synergetic approach can be taken into consideration as a fundamental method in treating intestinal dysbiosis in those patients resistant to other treatments.

Considering that intestinal dysbiosis is a real pathology capable of involving the entire Regulatory System, such as to underlie the multifactorial pathogenesis of numerous other pathologies, above all chronic (allergies, immune deficiency diseases, rheumatic diseases, cardiovascular diseases, headaches, neuroses, etc.), it seems particularly important to carry out a diagnostic evaluation by evaluating the indole and skatole in the urine, both, as a preventive measure, in subjects in apparent good health, and for therapeutic purposes, in patients affected by chronic diseases of an allergic and/or degenerative nature, caused or aggravated by intestinal dysbiosis. 
This observational study can therefore be a starting point for developing new intervention strategies based on the use of the oxygen-ozone mixture, with a view to general improvements in the state of health of modern Western society, increasingly afflicted by this pathology, which is only the tip of the iceberg of a long series of symptoms and disorders that afflict it because it is based on eating habits that are anything but healthy.

In conclusion, the study confirmed the validity of the treatment with ozonated water, combined with the rectal insufflation of the oxygen and ozone mixture, in controlling symptoms linked to dysbiosis and promoting restoration of correct environmental homeostasis in adults' intestinal microclimate.

Another element of chronic inflammation and intestinal dysbiosis can be traced back to stress, which is a general Adaptation Syndrome designed to re-establish a new internal balance (homeostasis) following stress factors (stressors). Stress is the psychophysical response to a number of emotional, cognitive or social tasks perceived by the person as excessive. Changes in internal balance can occur at the endocrine, humoral, organic and biological levels.

The term stress was first introduced into biology by Walter Cannon in 1935; the syndrome was defined in this way by Hans Selye in 1936.

The syndrome may be physiological, but it may also have pathological implications, including chronic ones, which fall within the field of psychosomatics. In general we usually distinguish between eustress and distress, or good stress that leads to solving problems and bad stress.

In general, the responses following exposure to stressors can be grouped in two ways:

i) Instant responses, due to the nature and physiology of the nervous system (e.g. sympathetic nervous system which uses already synthesised and stored neurotransmitters);

ii) Delayed responses, due to the nature and physiology of the endocrine and neuroendocrine system (e.g. reception of the stressor, activation of transcription of hormone-synthesising proteins, synthesis of hormones and release of these into the humoral circle).

Although the responses are different according to different stressors, the response mechanism is stereotypical and involves, in series:

i) First an instant response, by stimulating the sympathetic system - adrenal medulla;

ii) Subsequently a delayed response, through the action of the hypothalamic-pituitary-adrenal axis).

The signalling mechanism between one anatomical structure and the other also defines the response speed. For example, the fast response is based on neurotransmitters (fast to synthesise and easy to release because they are stored in vesicles). The slow response is based on hormones. Unlike neurotransmitters, hormones are produced only after a long time (hours or days).

If the stressors are particularly intense and prolonged, the body goes through several phases:

i) Alarm phase: in which biochemical and hormonal modifications of immediate adaptation take place;

ii) Resistance phase: in which the organism organises itself to protect itself as a whole;

iii) Exhaustion phase: in which the defences are lowered and the body is unable to maintain homeostasis.

If this third phase is reached, the pathological state of stress persists, manifested through various symptoms: fatigue, heaviness of the limbs, cramps, insomnia, change in sensory perceptions, migraines, anxiety, tachycardia, apathy, depression, lowered immune defences, and many more.
A situation of chronic stress, in a subtle and sometimes silent manner, can lead to a state of chronic inflammation and acidosis of the extra-cellular matrix.

Very often there are no obvious clinical manifestations and they can take on a chronic, subtle course, representing one of the main causes of many chronic degenerative diseases (such as heart attacks, autoimmune diseases, chronic bronchitis, cerebral stroke, type 2 diabetes, hypertension, multiple sclerosis, Alzheimer's disease, and many others right up to neoplasms); this is in relation to the increase in circulating glucocorticoids, the loss of their circadian rhythm, and excessive activation of the sympathetic nervous system.

Intestinal bacteria altered as a result of inflammatory bowel diseases, which can lead to all these disparate diseases, are increasingly being studied in Italy and abroad. In Italy, a group of Swiss and Italian scientists working at the IRCCS Fatebenefratelli in Brescia have identified pro-inflammatory microbes in the intestine that could be the cause of Alzheimer's disease.

A further study conducted by the University of Lund in Sweden, to study the composition of intestinal bacterial flora for Alzheimer's disease research, has shown that intestinal bacteria can accelerate the development of this disease.

The results open the way to new opportunities for disease prevention and treatment. Intestinal bacteria have an important impact on our health through the interaction between the immune system, the intestinal mucosa and our diet.

By studying healthy and sick mice, the researchers discovered that mice with Alzheimer's disease have a different composition of intestinal bacterial flora than healthy mice.

The researchers also studied Alzheimer's disease in mice that were completely devoid of intestinal bacteria, to further test the relationship between intestinal bacteria and the disease, and found that mice without bacteria had a significantly lower quantity of betaamyloid plaques in the brain, the hallmark of Alzheimer's disease.

To clarify the link between intestinal flora and the onset of the disease, the researchers transferred the intestinal bacteria of sick mice to bacteria-free mice, and found that mice which received intestinal bacteria from sick mice developed more beta-amyloid plaques in the brain compared to mice that received intestinal bacteria transferred from healthy mice.

Researcher Frida Fak Hallenius, from the Food for Health Science Centre at Lund, has reiterated the uniqueness of their study, as it demonstrated a direct causal link between intestinal bacteria and Alzheimer's disease. The clear and irrefutable demonstration that mice which completely lacked bacteria developed fewer beta-amyloid plaques in the brain.

She states that: "The results indicate that we can now begin to look for new ways to prevent the disease and delay its onset. We believe this is an important step forward, as we are currently only able to alleviate the symptoms of Alzheimer's disease with antiretroviral drugs."

The research is the result of an international collaboration between Professor Frida Fak Hallenius and doctoral student Nittaya Marungruang from the Food for Health Science Centre at Lund and a research group at the École Polytechnique Fédérale in Lausanne, Switzerland. The collaboration has now expanded and includes researchers from Germany and Belgium.

Researchers continue to study the role of intestinal bacteria in the development of Alzheimer's disease and test new types of preventive and therapeutic strategies based on the modulation of intestinal bacterial flora, through diet and new types of probiotics.

Other studies have been done on transgenic mice, which express the amylogenic fragment of the amyloid beta precursor protein in Alzheimer's disease. These mice were producers of extracellular amyloid beta, and showed an increase in glycolysis 
and microglial activity, also producing very damaging free radicals, cellular loss in the hippocampus and development of spatial learning deficits. It was also found that modification of the microbiota influenced the development of cerebral amyloid beta.

Increasing evidence suggests that the gastroenteric tract is the bridge between Microbiota and the Central Nervous System (CNS): Psychobiotics is a new, very recent discipline that deals with studying the system of relationships between mind-bodymicrobiota-diet.

The interconnection between mental health and intestinal microbiota is increasingly being studied.

Now that we know that intestinal bacteria (that same intestine being our second brain) can speak to the CNS, influencing our mood, our appetite, the functioning of our thinking and even our circadian rhythms, the next challenge for doctors and researchers will be to monitor this communication.

While it has been known for more than a century that bacteria can have positive effects on physical health, only in the last 10-15 years have we been able to observe signals that have shown there is a connection between the intestine and the brain.

Experimental animal studies have shown that improved immune function, better stress reactions and even some learning and memory benefits have been attributed to adding the right strains of bacteria to the intestinal microbiome.

In 2017, Harac et al. ${ }^{14}$ provided very convincing data on the consistency of the relationship between Human Intestinal Microbiota and the development of Alzheimer's disease, the most widespread form of dementia in the Western world.

The researchers found that the abundance of at least two major bacterial strains, firmicutes and bacteroides, in the faecal microbiota, was significantly altered in transgenic mice that develop greater amyloid plaques: at the genetic level, allobaculum and akkermansia muciniphila decreased while the strains of rikenellaceae and S24-7 increased.

Low levels of akkermansia muciniphila in the microbiota coincide with the development of obesity and type 2 diabetes in the mouse, and the restoration of akkermansia muciniphila in the intestine results in a reduction in fat mass and a decrease in systemic inflammation.

On the other hand, type 2 diabetes and inflammation are risk factors for Alzheimer's disease. The results from Harac et al. ${ }^{17}$ further support the emerging view that alterations of the Microbiota may contribute to the development of a wide range of neurological and neurodegenerative diseases far beyond metabolic syndrome, diabetes and obesity.

The theory of free radicals in the etiopathogenesis of senile dementia is scientifically accepted, and based on this, various antioxidant drugs such as Vitamin E, selegiline and Ginkgo Biloba extract have long been used as an adjuvant treatment. However, none of these have demonstrated long-term benefits in these patients. Ozone is a modulator of biological oxidative stress. The production of reactive oxygen species, including hydroxyl free radicals $(\mathrm{OH})$, superoxide anion $\left(\mathrm{O}_{2}\right)$ and other non-radicals such as hydrogen peroxide or hypochlorous acid (HCIO), is part of normal biological metabolism and also necessary for a series of life processes such as phagocytosis; however, the uncontrolled increase in these reactive species and free radicals is directly related to a wide variety of pathogeneses, including Alzheimer's and Ageing. The control of reactive oxygen species and free radicals in the body corresponds to antioxidant enzyme systems, mainly the enzymes superoxide dismutase, catalase and glutathione peroxidase.

Therefore, an excess of free radicals or the failure of these protection systems to counteract overproduction inevitably leads to multiple pathological processes.
There are mechanisms associated with signal transduction that activate or repress transcription of specific genes responsible for modulating oxidative stress.

Ozone has the property of stimulating certain protective antioxidant enzyme systems against oxygen metabolites. Therefore, ozone therapy generates a dynamic regulation between the pro-oxidant activity and the antioxidant activity generated by the organism, which maintains the structure of the membrane and cellular metabolism.

In addition to antioxidant activity, ozone stimulates the transport mechanisms and the use of oxygen at the cellular level.

And finally, ozone has been shown not to cause adverse reactions.

Ozone therapy is an effective therapeutic procedure in managing elderly people with dementia, especially as regards their autonomy, which provides better consistency, as well as in the activities of daily life, particularly in mixed multi-infarct and senile dementia.

The treatment can exploit various ways synergistically: GAE, drinking hyperozonated water, rectal insufflation.

For some years, study has been taking place of the use of ozone in agriculture and cattle breeding. A subject that has become increasingly topical after the international attention to the use of Glyphosate.

Glyphosate is considered a carcinogen by the WHO but still used in Europe, along with other chemical pesticides. In Italy, however, a national plan has been approved for the biological plan and the use of natural fertilisers such as Biozon Professional, based on ozone and anti-parasitic agent.

As mentioned, there is dysbiosis with food intolerances: to lactose and gluten, which are actually an indicator of the human body's response to the use of chemical herbicides and pesticides.

Glyphosate and other chemical pesticides have also been found, in various clinical studies, to damage the health of adults and children.

A study on glyphosate by the researcher Caroline Cox illustrated the possible effects on human health: increase in oestrogenrelated breast cancers, thyroid cancer, colon cancer, testicular cancer, myelomas, non-Hodgkin's lymphomas, increase in cases of autism.

In the US, according to the Environmental Protection Agency the use of this agricultural herbicide has exceeded 750 million $\mathrm{kg}$, and even in Italy the figures remain high, with consumption of approximately $55,000 \mathrm{~kg}$. Little or nothing is known about use in other countries. Its main use is in the cultivation of wheat, the maturation of which is accelerated through treatment with glyphosate, allowing quick drying and producing flours which are harmful to health, with about $12-15 \%$ more gluten, causing increases in celiac disease and metabolic disorders.

In March 2015, the International Agency for Research on Cancer (IARC) had defined the substance as a carcinogen and classified it as probably carcinogenic to humans. According to a recent report by the World Health Organization, the devastating effects of chemical pesticides and herbicides are the primary cause of death by poisoning for at least 200,000 people.

In Italy, a law limiting its use in Italy and in trade, with the exception of private use, came into force on 22 August 2016.

Conversely, the European Commission continues to extend its strong use of the statements from a study by the European Chemicals Agency (ECHA), whose research claims that "glyphosate is not carcinogenic and does not cause genetic mutations but only serious eye damage, and is toxic with long-lasting effects on life in aquatic environments".

Greenpeace responded by stating that "the ECHA has deliberately ignored scientific evidence on the correlation between the use 
of this herbicide and some forms of cancer", precisely to favour the commercial interests of some chemical giants, ensured through lobbying.

The legislative decrees for pasta and rice have finally entered into force, with the obligation to indicate the origin of the raw materials on the label.

Measures that large companies have tried to oppose, but Italy, fortunately, has chosen to carry on, despite the fact that some predict the risk of the European Union stopping it. The new labels show the country of cultivation and that of milling for wheat, and that of cultivation, processing and packing for rice.

It is undoubtedly a significant step forward towards full recognition of the consumer's sacrosanct right to be adequately informed about the origin of the food they put on the table. Add to this the fact that our country - unlike others such as Canada, from which we import large quantities of wheat - offers additional guarantees in terms of food safety, such as prohibiting the use of glyphosate.

However, the goal cannot just be protecting individual producers of Italian rice and wheat, making their products more profitable as a whole.

A narrative label, more transparent, should report not only information on the origin but also an accurate and comprehensible description of the entire production process.

Italy, in contrast, has acknowledged the serious health risks of this herbicide, after having traced a sizable percentage of it in mass-produced pasta and bread, and proposes a ministerial plan in favour of increasingly natural and sustainable agriculture that respects both the environment and consumer health. It is precisely in this new ecological and biological perspective that BIOZON F1 is introduced, an invigorating and potentially ozone-based solution with an antiparasitic and invigorating function, which replaces chemical pesticides thanks to an immunostimulatory mechanism, which makes the plant immune from external attacks and more flourishing in appearance. The ozonated solution for agricultural purposes originates from three oxygen molecules, which react with each other to give rise to ozone, a naturally occurring gas with very powerful antibacterial, antifungal and antioxidant properties. The gaseous ozone thus reacts with the oily base, incorporating itself in the liquid and thus keeping its valuable characteristics unaltered. The invigorating function of this valuable $\mathrm{O}_{2}-\mathrm{O}_{3}$ compound has been scientifically tested in the study by the Agricultural Research Council.

The test showed that Biozon Professional is able to improve the responses of plants subjected to biotic and abiotic stress, increase the number of secondary metabolites in plant tissues, chlorophyll and polyphenol concentrations. A natural product that respects the ecosystem and the health of the global population, for healthy fruit and vegetable production free of fungicides, in accordance with the new European and global directives: a first step towards combating the many diseases that originate from environmental contamination.

Interest in the application of oxygen-ozone is ever-increasing in agriculture, as well as in the zootechnical field, to allow a more natural and respectful approach to the product, but also one that is more convenient for producers.

Italian legislation also recognised its validity in 1966, with Protocol No. 24482 from the Ministry of Health, which considers ozone "a natural defence for sterilising environments", thus leading to its use in the health, livestock, agribusiness, military and civil sectors.

In the fruit and vegetable sector, the use of ozone in cold storage, through a system for injecting gas into the air, allows a delay of fruit and vegetable expiry dates, the control of odours and the development of ethylene, naturally generated from products in a state of ripeness.

To disinfect the processing and packaging lines. To disinfect and recycle the washing water.

To eliminate listeria, salmonella and other yeasts, bacteria, viruses and moulds. To eliminate insects.

Long-term tests and experience have shown that washing fruit and vegetables with ozonated water increases the product's storage capacity, reduces costs and eliminates pesticides, ammonia and other unwanted substances that may be present on the crops.

This application, in addition to the benefits for product protection and hygiene, allows natural fruit ripening to be delayed, with positive effects on colour, texture and weight.

In the production of wines and other beverages like beer, fruit juices, preserves and mineral water, ozone can be used for: i) sanitisation of filling and bottling lines; ii) sanitisation of tanks and storage tanks; iii) disinfecting bottles before bottling.

In the dairy sector, ozone can be used to disinfect, sterilise and obtain adequate environmental control in milk and cheese packaging and preparation plants, and for sanitising water for mozzarella and dairy products. Water treated with ozone is in fact free from unpleasant smells and tastes, and plays an important germicidal and abatement action on all organic micropollutants. Ozone, used in the maturation rooms, makes it possible to obtain products without unwanted moulds (green, yellow, black) in a practically sterile environment with pure air. It is also used on cheeses such as ricotta, gorgonzola and taleggio to fight bacteria, and in various cheese curing chambers to avoid insect infestations and parasites. Furthermore, ozone can be used in the treatment of whey and sewage sludge to reduce disposal costs.

For years, ozone has been successfully used by numerous companies in processing and packaging fish products. Ozone can in fact be used effectively in the following industrial phases:

i) in the washing and processing phases for fish. In these phases, ozonated water can be used to reduce odours, disinfect the fish, increase the storage capacity by several days and reduce the formation of ammonia deriving from putrefaction processes, avoiding leaving residues such as chlorine;

ii) in the purification phases of filter feeders (such as, for example, mussels, clams, cockles, etc.) and in the steps following their harvesting;

iii) during the washing and external cleaning phases of these products, before their final entry into the commercial circuit.

Ozone can be used in incoming or recirculated water in farms, obtaining a marked improvement in the quality of the water itself: Reducing bacteria, viruses, protozoa and pathogenic fungi in fish. Eliminating colloidal substances suspended in water and removing dissolved organic substances that can stress fish.

Eliminating ammonia and nitrites that can be toxic to fish. Greater speed in fish growth.

Greater fish production obtainable with the same facilities. Fish product obtained at a lower cost.

In beekeeping it is possible to use ozone with the aim of increasing the quality of honey harvests and, at the same time, improving the life cycle of the bees. In fact, by using ozone:

It fights the varroa destructor, an external parasitic mite that attaches itself to bees and weakens them, sucking their vital fluids.

The premises and facilities used for honey extraction are protected against viruses, bacteria, moulds and yeasts. It acts against moths and other insects that can proliferate in hives, compromising the quality of honey and beeswax.

Another field where studies are being carried out is that of assessing how ozone's characteristics are usable and sustainable in the greenhouse sector. 
The first, very promising results were obtained in greenhouses by spraying ozonated water ( $6 \mathrm{ppm})$ at high power, used for spraying the solution itself in these experimental sites.

In greenhouses, thanks to the devitalising action on spores and mycelia of saprophytic and parasitic fungi, it can be used without shortage.

The action of activated oxygen (ozone) in disinfecting environments and devitalising phytopathogenic microorganisms and harmful insects, thanks to its very high oxidising power, is technically acquired and incontrovertible data at the international level.

It is important to know that ozone, an unstable gas, at $20^{\circ} \mathrm{C}$ has a half-life of 2-3 days depending on the humidity and temperature onsite, while in aqueous solution the persistence time and oxidative effectiveness are reduced to just 15-20 minutes. This aspect is very important for users, who cannot store ozone but must produce it at the time of use. The main mechanism of action is lipid peroxidation which causes damage to the membrane phospholipids of microorganisms. The toxicity of activated oxygen also depends on its ability to oxidise amino acids, irreversibly altering proteins' structure and function.

The strategic importance of activated oxygen lies in the fact that, due to its oxidising and antimicrobial activity, it is transformed into atmospheric oxygen. The activated oxygen therefore leaves no residue either in the environment or on the treated vegetation.

Thus the ozone, killing without leaving a trace, is a perfect killer; just a few minutes after applying it on the plants, you can proceed to harvest each product without any safety interval limit. This is an aspect of fundamental importance for farmers, who are always struggling with the restrictions on using crop protection products.

A few minutes after applying it on the affected vegetation, the results of ozone's devitalising action on germs, spores and mycelia of saprophytic fungi and parasites can occur.

Ozone can be released as a gas or dissolved first in water and then sprinkled or sprayed on crops, killing non-selectively and only by contact. In fact, it precludes any devitalising effect due to absorption and translocation within cells and tissues.

With the ozonated Biozon F1 oil, it is possible to eradicate parasitic infections. It can be used by diluting the product and distributing it through an atomiser.

In zootechnics, using oxygen-ozone reduces the use of drugs such as antibiotics and antibacterials by up to $60-70 \%$.

$\mathrm{O}_{3}$ generator systems are used. Ozone is mixed with water for best disinfection and to make both the animals' nourishment and watering healthier; another plant can be used to purify the air and inject oxygen-ozone into the stalls through a system of ducts on the ceiling, annihilating the germs in the air and preventing their transmission from animal to animal, improving their intestinal flora and increasing immune defences. A significant reduction in respiratory diseases and a large decrease in the use of antibiotics and disinfectants.

This leads to a reduction in the cost of treating infectious diseases, cleaning shelters, containing odours, buying acids and the animals' mortality, making the meat healthier and improving the animals' living conditions.

The economic advantages are also indisputable: there is an increase in exports to those countries, such as Italy, which are very careful regarding the purity of the product and the concentration of antibiotics. In fact the increased bacterial resistance to antibiotics found in humans is quite similar to that of animals. One of the most urgent problems for pig, poultry, sheep and cattle farmers is in fact the increasingly high consumption of chemicals and antibiotics to fight infections, viruses and diseases that threaten farmed animals. Along with the increasing costs, there is a worrying concentration of these substances in the slaughtered, sold and consumed meat, a consequence of the fact that even minor infectious diseases are overcome not with improved breeding techniques but with the use of antibiotics.

A fact that is not insignificant, considering how antibiotic resistance is an increasingly concrete problem not only in animals but also in humans, to the point of being recognised as a global health emergency by the World Health Organization. Precisely for this reason, we need to learn more about the possibility of using alternative medical approaches, such as ozone therapy, where it is not necessary to resort to antibiotics.

University education places great importance on treatment strategies with chemical molecules, but little on the immune system, and the use of natural medicine is very limited.

Thus the mentality and habits of doctors are formed through prescribing medicines that have long been effective and practical in administration. In veterinary medicine the problem has taken place along the same lines as human medicine, with the aggravating result of decreasing the healthiness of meat, eggs and milk. It is a consequence of too many antibiotic residues in the products in our diet.

Such treatments can also help increase antibiotic resistance in humans: consider that as much as $70 \%$ of the antibiotics produced in the world are used in the food industry.

There is no denying that the pharmaceutical industry has never disdained the large-scale and straightforward use of chemical medicines to treat infectious diseases in animals and humans.

\section{References}

1. Di Paolo N, Bocci V, Gaggiotti E. New aspects of oxygenozone therapy. Int J Artificial Organs 2004;27:168-75.

2. Bocci V. Biological and clinical effects of ozone. Has ozone therapy a future in medicine? Br J Biomed Sci 1999;56:270-9.

3. Bocci V. Does ozone therapy normalize the cellular redox balance? Implications for therapy of human immunodeficiency virus infection and several other diseases. Med Hypotheses 1996;46:150-4.

4. Clavo B, Pérez JKL, Lòpez L, et al. Effect of ozone therapy on muscle oxygenation. J Alternative Complement Med 2003; 9:251-6.

5. Shoemaker JM. Ozone Therapy - History, Physiology, Indications, Results. 2005. Available from: www.fullcircleequine.com/oz therapy.pdf

6. Bocci V. Ossigeno Ozonoterapia. Comprensione dei meccanismi d'azione e possibilità terapeutiche. Milano: Casa Editrice Ambrosiana; 2000.

7. AA.VV. Why consider ozone therapy/oxygen Spa as alternative treatment? 2010. Available from: http//www.holisticbodyworker. com/ozone therapy documentation.html

8. Bocci V. Ozonization of blood for the therapy of viral disease and immunodeficiencies. A hypothesis. Med Hypot 1992;39:30-4.

9. Sharma M, Hudson JB. Ozone gas is an effective and practical antibacterial agent. Am J Infect Control 2008;36:559-63.

10. Loprete F, Franzini M. Ozono vs Disbiosi. Bergamo, Italy: SIOOT Publications; 2015.

11. Loprete F, Vaiano F. The use of ozonated water and rectal insufflation in patients with intestinal dysbiosis. Ozone Ther 2017;2:56-61.

12. Vaiano F, Loprete F. Large auto-hemoinfusion versus rectal insufflation in patients with metabolic syndrome. Ozone Ther 2016;1:13-6. 
13. Bocci V, Zanardi I, Huijberts MS, Travagli V. Diabetes and chronic oxidative stress. A perspective based on the possible usefulness of ozone therapy. Diabetes Metab Syndr 2011;5:45-9.

14. Harach T, Marungruang N, Duthilleul N, et al. Reduction of Abeta amyloid pathology in APPPS1 transgenic mice in the absence of gut microbiota. Sci Rep 2017;7:41802.

15. De Vera GA, Stalter D, Gernjak W, et al. Towards reducing DBP formation potential of drinking water by favouring direct ozone over hydroxyl radical reactions during ozonation. Water Res 2015;87:49-58.

16. Pistollato F, Sumalla Carro S, Elio I, et al. Role of gut micro- biota and nutrients in amyloid formation and pathofenesis of Alzheimer disease. Nutr Rev 2016:74:624-34.

17. Jiaing $\mathrm{C}$, Li G, Huang $\mathrm{P}$, et al. The Gut microbiota and Alzheimer's disease. J Alzheimers Dis 2017;58:1-15.

18. Hu X, Wang T, Jin F. Alzheimer's disease and Gut microbiota. Sci China Life Sc 2016;59:1006.

19. Liu X, Cao S, Zhang X. Modulation of Gut Microbiota-Brain axis by probiotics, prebiotics, and Diet. J Agric Food Chem 2015;63:7885-95.

20. Friedland RP, Chapman MR. The role of microbial amyloid in neurodegeneration. PLoS Pathol 2017;13:e1006654. 